\title{
Orthostatic Reactivity in Patients with Diabetic Neuropathy
}

\author{
Daniela Lubenova ${ }^{1}$, Ekaterina Titianova² ${ }^{2}$ Dance Vasileva $^{3^{*}}$ \\ ${ }^{1}$ National Sports Academy "V. Levski" - Department of Kinesitherapy and Rehabilitation, Sofia, Bulgaria; ${ }^{2}$ Military Medical \\ Academy - Department of Neurology and Neurosurgery, Sofia, Bulgaria; "University "Goce Delchev" - Faculty of Medical \\ Sciences, Stip, Macedonia
}

\begin{abstract}
Citation: Lubenova D, Titianova E, Dance Vasileva D. Orthostatic Reactivity in Patients with Diabetic Neuropathy. OA Maced J Med Sci. 2014 Jun 15; 2(2):244-248. http://dx.doi.org/10.3889/oamjms.2014.039

Key words: Exercise Therapy; Orthostatic Tolerance; Physical Therapy; Type II Diabetes; Kinesitherapy.

Correspondence: Miss Dance Vasileva. University "Goce Delchev", Faculty of Medical Sciences, Krste Misirkov no. 10-A, Stip 2000 Republic of Macedonia. Sip dance.vasileva@ugd.edu.mk

Received: 10-Mar-2014; Revised: 16-Mar2014; Accepted: 01-May-2014; Online first: 12-May-2014

Copyright: ๑ 2014 Lubenova et al. This is an open access article distributed under the terms of the Creative Commons Attribution License, which permits unrestricted use, distribution, and reproduction in any medium, provided the original author and source are credited.

Competing Interests: The authors have declared that no competing interests exist.
\end{abstract}

\section{Abstract}

AIM: The purpose of the study was to assess the effect of a structured physical therapy (PT) programme on the orthostatic reactivity in patients with diabetic neuropathy (DNP).

MATERIAL AND METHODS: The study was performed in 90 patients with DNP (34 male and 56 female, mean ages $60.8 \pm 7.8$ years) of lower extremities. The orthostatic autoregulation was evaluated using an active orthostatic test. The arterial blood pressure and the heart rate were determined after 10 minutes of rest in lying position before and after 1, 5 and 10 minutes of active standing.

RESULTS: At the start of the study a normotonic orthostatic reactivity (NOR) was observed in 32 patients. Abnormal sympathicotonic type of orthostatic reactivity (SOR) was found in 18 patients and asympaticotonic type of orthostatic reactivity (AOR) was established in the remaining 40 patients. After the PT treatment a significant improvement of the orthostatic autoregulation in the groups with SOR and AOR was not found - NOR was observed in 66 patients with DNP (80.3\%) at 6 weeks after the start of PT.

CONCLUSION: The applied structured PT, later continued as a home exercise programme, significantly improved the orthostatic reactivity in patients with orthostatic dysregulation due to DNP.

\section{Introduction}

Diabetic neuropathy (DNP) is one of the most common complications in patients with diabetes mellitus type 2. It affects from $22.5 \%$ to $28.5 \%$ of all diabetic patients worldwide [1, 2]. The autonomic neuropathy is less frequent -from $5 \%$ to $7 \%$ of the diabetic patients. It depends on the duration of the diabetes and the age of the patient [2-4]. Cardiovascular autonomic neuropathy is a common form of diabetic autonomic neuropathy and causes abnormalities in heart rate, peripheral vascular dynamics and orthostatic tolerance $[1,2]$.

The orthostatic autoregulation in human appeared as an adaptive and compensatory mechanism against the gravitational redistribution of the blood in the transition from horizontal to upright body position. Postural changes in humans activate the gravity forces that lead to a rise in the intravascular pressure and redistribution of the blood to the lower extremities below the so-called "hydrostatic indifferent point" [4]. Gravitational forces stimulate a complex of compensatory mechanisms to preserve a stable cerebral hemodynamics. An important role for limiting the orthostatic gravitational blood redistribution plays the "peripheral muscle pump", i.e. the contraction of the calf muscles. Through the compression of the deep veins of the legs the blood returns to heart at the time of active standing and during physical exercise. The peripheral muscle pump insufficiency worsens the venous return to heart and contributes to the development of orthostatic intolerance and/or cerebral ischemia [5]. Similar insufficiency is observed in patients with diabetes mellitus and DNP of lower extremities, where a peripheral denervation is present, often combined with atrophy of the calf muscles. In such cases, signs of orthostatic intolerance may develop, which can aggravate the course of the disease. 
The role of the physical therapy (PT) in the treatment of DNP is well recognized, but its influence on the orthostatic reactivity of DNP patients is less studied.

The aim of the present study was to assess the effect of a structured PT programme on the orthostatic reactivity in patients with DNP.

\section{Subjects and Methods}

Ninety patients (34 male and 56 female, mean ages $60.8 \pm 7.8$ years, range $50-70$ years) with diabetes mellitus type 2 and DNP of lower extremities have been studied. The diabetes mellitus duration ranged from 1 to 18 years, mean duration $9.5 \pm 5.6$ years. At the time of the study 40 patients were treated with oral drugs, and the other 50 patients were on insulin therapy. The following concomitant diseases were present: arterial hypertension in 42 patients, cardiac diseases in 34 patients, obesity in 37 patients and retinopathy in 87 patients. All patients were capable for independent walking; they were without serious communication problems and gave their written informed consent to participate in the study.

Along with the neurological examination, performed in all patients, the circumference of the calf muscle was measured $(\mathrm{cm})$ and manual muscle testing (MMT) was performed of the dorsal leg flexors [6]. Using standard technique in electroneuromyography (ENMG) the sensory and the motor conductivity of the lower limbs were examined at the start of the study and 6 weeks after the beginning of the PT.

In all patients a parallel treatment with alphalipoic acid (600 mg daily as i.v. infusion for 7-10 days) along with a specialized intensive 45 minutes 10 days PT was performed. The daily physical therapy routine lasted 45 minutes and the intensity of exercise was moderate. In the introduction, the exercises were aimed at preparation of the body for the upcoming exercises, through gradual adaptation of the cardio-vascular system (thoracic and diaphragm breathing, rhythmic exercise for distal muscle groups, isometric exercise in circulatory pattern). In the main part the physical therapy included exercises to increase the muscle strength (through exercise against controlled resistance, given through elastic Tera-Bands), to improve sensation, coordination and equilibrium of the patients (exercises were tended to disturbances) and using bicycle ergometers to increase the adaptive capabilities of the cardio-vascular system to higher workloads. The closing part included exercises relaxation for the patients. At the end, the patients were instructed to continue to exercise at home following the instructions given by the physiotherapist.

The orthostatic autoregulation was evaluated using an active orthostatic test at the start of the study, at day 10 and 6 weeks after the beginning of the PT. The test was performed in the morning between 8 and 10 o'clock, at a room temperature of $21-23^{\circ} \mathrm{C}$. The heart rate $(\mathrm{HR})$, the systolic $(\mathrm{SBP})$ and the diastolic (DBP) blood pressure were taken after 1, 5 and 10 minutes of a lying position using a cuff method [5] and the values at 10 minutes were considered the baseline. Then the patients were actively stood up and the same measurements were performed after 1, 5 and 10 minutes of active standing. After that the patients were returned in a lying position and the registration procedure was repeated.

Based on the type of the orthostatic reactivity, following the classification of Thulesius [7], the patients were divided into 3 groups: normotonic orthostatic reactivity (NOR), where the heart rate $(\mathrm{HR})$ increased up to 20 beats per minute (bpm) and the SBP changes were no more than $10 \mathrm{mmHg}$ while standing; a pathological sympathicotonic orthostatic reactivity (SOR), where the HR increased more than $20 \mathrm{bpm}$ and the SBP dropped more than $10 \mathrm{mmHg}$; and an asympathicotonic orthostatic reactivity $(\mathrm{AOR})$, where no or minimal changes in the HR along with a decrease in the blood pressure were found.

\section{Statistical methods}

The data obtained were process statistically using variation and alternative analyses. Paired $t$-test was applied to compare the parameters before, at day 10 and 6 weeks after the PT. The non - parametric data of MMT test were analyzed by Wilcoxan test. $P$ value less than 0.05 was considered statistically significant.

\section{Results}

The clinical characteristics of the patients are presented in Table 1. The AOR patients were significantly older and had a longer duration of the diabetes mellitus compared with the other subgroups.

Table 1: Clinical characteristics of the patients with DNP.

\begin{tabular}{|c|c|c|c|}
\hline Parameters & $\begin{array}{l}\text { NOR } \\
(n=32) \\
\end{array}$ & $\begin{array}{l}\text { SOR } \\
(n=18)\end{array}$ & $\begin{array}{l}\text { AOR } \\
(n=40)\end{array}$ \\
\hline Mean age (years) & $58.9 \pm 7.4$ & $59.5 \pm 7.9$ & $62.9 \pm 8.5^{*}$ \\
\hline Male/female & $14 / 18$ & $8 / 10$ & $12 / 28$ \\
\hline Diabetes duration (years) & $6.5 \pm 6.3$ & $6.8 \pm 4.3$ & $15.3 \pm 6.1^{* \star *}$ \\
\hline
\end{tabular}

The results from the calf circumference measurements, the MMT of the dorsal flexors and the nerve conduction velocity of the lower extremities are shown in Table 2.

A significant enlargement of the calves of all patients was found; more pronounced 6 weeks after the treatment. These data were associated with an increase in dorsal flexors muscle strength, evaluated by MMT, significant in the NOR, SOR and AOR groups. Additionally, an improvement was observed in the latency times for the sensory and the motor conductions of the lower limbs 6 weeks after the PT. 
Table 2: Mean values and standard deviations of the selected parameters in patients with DNP according to their type or orthostatic reactivity.

\begin{tabular}{|c|c|c|c|c|c|c|c|c|c|c|}
\hline \multirow{2}{*}{ Parameters } & \multirow{2}{*}{ Side } & \multicolumn{3}{|c|}{ NOR } & \multicolumn{3}{|c|}{ SOR } & \multicolumn{3}{|c|}{ AOR } \\
\hline & & At start & Day 10 & 6 Week & At start & Day 10 & 6 Week & At start & Day 10 & 6 Week \\
\hline Circumference of the & Left & $37.3 \pm 2.8$ & $37.8 \pm 2.8$ & $38.2 \pm 2.9^{\star}$ & $37.9 \pm 1.5$ & $38.4 \pm 1.5$ & $39.0 \pm 1.5^{*}$ & $36.9 \pm 2.3$ & $37.4 \pm 2.5$ & $37.9 \pm 2.4^{*}$ \\
\hline calf muscles $(\mathrm{cm})$ & Right & $37.5 \pm 2.8$ & $37.9 \pm 2.8$ & $38.3 \pm 2.9^{*}$ & $38.5 \pm 1.9$ & $38.91 \pm 1.8$ & $39.3 \pm 1.7^{\star}$ & $37.3 \pm 2.4$ & $37.7 \pm 2.5$ & $38.3 \pm 2.4^{*}$ \\
\hline MMT of the lower & Left & $4.6 \pm 0.5$ & $4.9 \pm 0.3$ & $5.0 \pm 0.0^{*}$ & $4.7 \pm 0.5$ & $4.9 \pm 0.3$ & $5.0 \pm 0.0^{*}$ & $4.5 \pm 0.5$ & $4.7 \pm 0.5$ & $4.9 \pm 0.3^{*}$ \\
\hline extremities (score) & Right & $4.8 \pm 0.4$ & $5.0 \pm 0.0^{*}$ & $5.0 \pm 0.0^{*}$ & $4.4 \pm 0.5$ & $4.5 \pm 0.5$ & $5.0 \pm 0.0^{\star}$ & $4.5 \pm 0.5$ & $4.7 \pm 0.5$ & $4.9 \pm 0.3^{*}$ \\
\hline ENMG latency time & SC & $3.9 \pm 1.8$ & & $3.0 \pm 1.7^{\star}$ & $3.3 \pm 1.4$ & & $2.5 \pm 1.0^{\star}$ & $3.6 \pm 0.9$ & & $2.9 \pm 0.9^{\star \star}$ \\
\hline (ms) & MC & $4.8 \pm 0.9$ & & $3.8 \pm 0.9^{* *}$ & $4.8 \pm 1.1$ & & $3.5 \pm 1.0^{*}$ & $5.1 \pm 1.6$ & & $4.5 \pm 0.9^{*}$ \\
\hline
\end{tabular}

The results from the active orthostatic test performed in the three groups before the treatment, at day 10 and 6 weeks after the PT are summarized in Table 3. The significant changes in these parameters are presented in Figure 1.
In comparison to the baseline values the patients in the NOR group did not show significant changes in the haemodynamic parameters after the PT. The HR and the blood pressure remained relatively stable during the follow-up studies at day 10 and after 6 weeks.

Table 3: Orthostatic changes in patients with DNP according to their type or orthostatic reactivity before and after the structured PT.

\begin{tabular}{|c|c|c|c|c|c|c|c|c|c|c|c|}
\hline \multirow{4}{*}{$\begin{array}{l}\text { Parameters } \\
\text { Heart rate } \\
(\mathrm{bm} / \mathrm{s})\end{array}$} & \multirow{3}{*}{$\begin{array}{l}\text { Position } \\
\text { Laying }\end{array}$} & \multirow[b]{2}{*}{ Minutes } & \multicolumn{3}{|c|}{ NOR } & \multicolumn{3}{|c|}{ SOR } & \multicolumn{3}{|c|}{ AOR } \\
\hline & & & At start & Day 10 & 6 Weeks & At start & Day 10 & 6 Weeks & At start & Day 10 & 6 Weeks \\
\hline & & 1 & $70 \pm 6$ & $72 \pm 7$ & $72 \pm 6$ & $75 \pm 6$ & $74 \pm 6$ & $78 \pm 8$ & $75 \pm 8$ & $75 \pm 8$ & $74 \pm 8$ \\
\hline & position & 5 & $70 \pm 6$ & $71 \pm 7$ & $70 \pm 5$ & $73 \pm 6$ & $73 \pm 8$ & $75 \pm 4$ & $73 \pm 9$ & $73 \pm 7$ & $72 \pm 8$ \\
\hline & & 10 & $70 \pm 6$ & $68 \pm 6$ & $69 \pm 5$ & $73 \pm 6$ & $72 \pm 9$ & $73 \pm 5$ & $72 \pm 8$ & $73 \pm 7$ & $71 \pm 8$ \\
\hline & Active & 1 & $84 \pm 6$ & $84 \pm 8$ & $83 \pm 8$ & $102 \pm 6$ & $92 \pm 7$ & $89 \pm 9$ & $73 \pm 8$ & $77 \pm 7$ & $79 \pm 8$ \\
\hline & standing & 5 & $80 \pm 6$ & $80 \pm 10$ & $79 \pm 9$ & $92 \pm 6$ & $85 \pm 5$ & $85 \pm 8$ & $73 \pm 9$ & $76 \pm 8$ & $76 \pm 6$ \\
\hline & & 10 & $79 \pm 8$ & $77 \pm 7$ & $77 \pm 8$ & $94 \pm 7$ & $85 \pm 6$ & $84 \pm 5$ & $73 \pm 9$ & $73 \pm 6$ & $76 \pm 5$ \\
\hline & Laying & 1 & $72 \pm 5$ & $74 \pm 7$ & $72 \pm 8$ & $84 \pm 8$ & $80 \pm 7$ & $80 \pm 7$ & $73 \pm 8$ & $72 \pm 8$ & $71 \pm 6$ \\
\hline & position & 5 & $70 \pm 6$ & $68 \pm 8$ & $70 \pm 7$ & $75 \pm 11$ & $75 \pm 11$ & $74 \pm 8$ & $72 \pm 9$ & $79 \pm 8$ & $69 \pm 5$ \\
\hline & & 10 & $69 \pm 4$ & $68 \pm 7$ & $69 \pm 6$ & $75 \pm 8$ & $73 \pm 9$ & $72 \pm 7$ & $71 \pm 8$ & $69 \pm 7$ & $68 \pm 5$ \\
\hline SBP & Laying & 1 & $126 \pm 13$ & $125 \pm 16$ & $123 \pm 16$ & $141 \pm 16$ & $131 \pm 12$ & $124 \pm 11$ & $136 \pm 15$ & $138 \pm 16$ & $133 \pm 14$ \\
\hline \multirow[t]{8}{*}{$(\mathrm{mm} \mathrm{Hg})$} & position & 5 & $124 \pm 14$ & $123 \pm 17$ & $121 \pm 16$ & $136 \pm 15$ & $128 \pm 12$ & $120 \pm 4$ & $132 \pm 14$ & $133 \pm 16$ & $128 \pm 13$ \\
\hline & & 10 & $124 \pm 15$ & $122 \pm 15$ & $118 \pm 18$ & $134 \pm 11$ & $127 \pm 12$ & $120 \pm 7$ & $130 \pm 14$ & $131 \pm 15$ & $126 \pm 11$ \\
\hline & Active & 1 & $128 \pm 13$ & $128 \pm 9$ & $124 \pm 19$ & $130 \pm 16$ & $129 \pm 12$ & $120 \pm 11$ & $124 \pm 14$ & $128 \pm 16$ & $131 \pm 16$ \\
\hline & standing & 5 & $127 \pm 16$ & $126 \pm 19$ & $125 \pm 17$ & $124 \pm 14$ & $129 \pm 15$ & $122 \pm 8$ & $126 \pm 17$ & $130 \pm 15$ & $129 \pm 14$ \\
\hline & & 10 & $126 \pm 16$ & $125 \pm 18$ & $123 \pm 16$ & $124 \pm 13$ & $130 \pm 13$ & $121 \pm 11$ & $124 \pm 15$ & $127 \pm 17$ & $129 \pm 12$ \\
\hline & Laying & 1 & $126 \pm 17$ & $124 \pm 15$ & $124 \pm 18$ & $136 \pm 13$ & $131 \pm 11$ & $122 \pm 11$ & $128 \pm 15$ & $130 \pm 17$ & $126 \pm 11$ \\
\hline & position & 5 & $124 \pm 17$ & $123 \pm 16$ & $120 \pm 16$ & $131 \pm 14$ & $128 \pm 13$ & $122 \pm 11$ & $127 \pm 14$ & $126 \pm 15$ & $124 \pm 12$ \\
\hline & & 10 & $120 \pm 15$ & $121 \pm 14$ & $118 \pm 14$ & $131 \pm 13$ & $126 \pm 11$ & $121 \pm 9$ & $124 \pm 13$ & $125 \pm 15$ & $121 \pm 10$ \\
\hline DBP & Laying & 1 & $77 \pm 10$ & $77 \pm 5$ & $78 \pm 8$ & $75 \pm 10$ & $75 \pm 10$ & $74 \pm 7$ & $74 \pm 11$ & $74 \pm 7$ & $74 \pm 7$ \\
\hline \multirow[t]{8}{*}{$(\mathrm{mm} \mathrm{Hg})$} & position & 5 & $77 \pm 10$ & $78 \pm 7$ & $76 \pm 8$ & $74 \pm 10$ & $74 \pm 10$ & $74 \pm 5$ & $73 \pm 10$ & $72 \pm 8$ & $71 \pm 8$ \\
\hline & & 10 & $78 \pm 8$ & $75 \pm 6$ & $74 \pm 9$ & $75 \pm 10$ & $75 \pm 10$ & $72 \pm 7$ & $70 \pm 9$ & $71 \pm 6$ & $72 \pm 8$ \\
\hline & Active & 1 & $77 \pm 9$ & $80 \pm 8$ & $80 \pm 9$ & $82 \pm 11$ & $82 \pm 11$ & $73 \pm 8$ & $72 \pm 14$ & $75 \pm 8$ & $76 \pm 9$ \\
\hline & standing & 5 & $74 \pm 12$ & $80 \pm 7$ & $78 \pm 6$ & $83 \pm 11$ & $83 \pm 11$ & $76 \pm 5$ & $72 \pm 10$ & $74 \pm 5$ & $74 \pm 5$ \\
\hline & & 10 & $74 \pm 10$ & $79 \pm 9$ & $78 \pm 7$ & $81 \pm 10$ & $81 \pm 10$ & $77 \pm 7$ & $74 \pm 9$ & $73 \pm 4$ & $72 \pm 7$ \\
\hline & Laying & 1 & $75 \pm 11$ & $78 \pm 7$ & $76 \pm 9$ & $81 \pm 8$ & $78 \pm 10$ & $70 \pm 7$ & $71 \pm 10$ & $72 \pm 8$ & $72 \pm 6$ \\
\hline & position & 5 & $75 \pm 10$ & $79 \pm 4$ & $74 \pm 7$ & $80 \pm 9$ & $79 \pm 10$ & $63 \pm 7$ & $71 \pm 8$ & $72 \pm 8$ & $72 \pm 7$ \\
\hline & & 10 & $74 \pm 10$ & $76 \pm 7$ & $75 \pm 7$ & $81 \pm 6$ & $76 \pm 10$ & $70 \pm 7$ & $70 \pm 9$ & $71 \pm 8$ & $69 \pm 7$ \\
\hline
\end{tabular}

$\mathrm{NOR}=$ normotonic orthostatic reactivity; SOR = sympathicotonic orthostatic reactivity; $\mathrm{AOR}=$ asympathicotonic orthostatic reactivity; $\mathrm{SBP}=$ systolic blood pressure; $\mathrm{DBP}=$ diastolic blood pressure. The significance is given in Figure 1.

In the SOR group, a significant change (toward normalisation) in the HR most evident at the first minute of active standing was found. Compared to the baseline data, a significant drop in the HR (from 29.0 to 20.0 bpm) was established at day 10 . Six weeks after the start of the PT the changes in the HR during active standing were similar to those observed in the NOR group. Similar findings were olso found for the SBP.

After the PT in the AOR group there was a tendency for an acceleration of the HR on day 10 that was significant after 6 weeks. Also a tendency to an increase in the SBP was seen.
The beneficial effect of the PT on the orthostatic reactivity in DNP patients was proved by the change in the percent ratio between the subjects with different types of orthostatic intolerance - a significant increase in the number of the patients with NOR was observed immediately after the end of the PT, reaching $80.3 \%$ from the patients with DNP after 6 weeks from the treatment (Figure 2). 


\section{Discussion}

The study reveals that the physical measures, sensory and motor nerve conduction and orthostatic reactivity all improved in patients with DNP who received PT intervention. In all patients, an increase in the calves' circumference and the muscle strength, assessed by MMT, was found most expressed in the NOR group. It has been demonstrated that the recovery of the muscle strength and the increase in the muscle mass of the calves in the patients with DNP provides an improvement in the venous influx and the systemic hemodynamics $[6,8,9]$.

a)

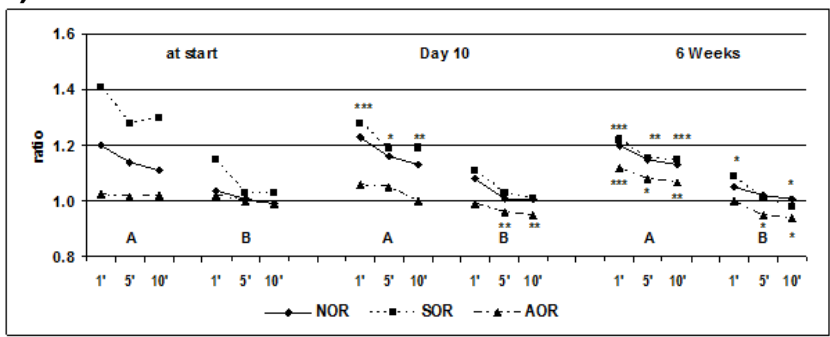

b)

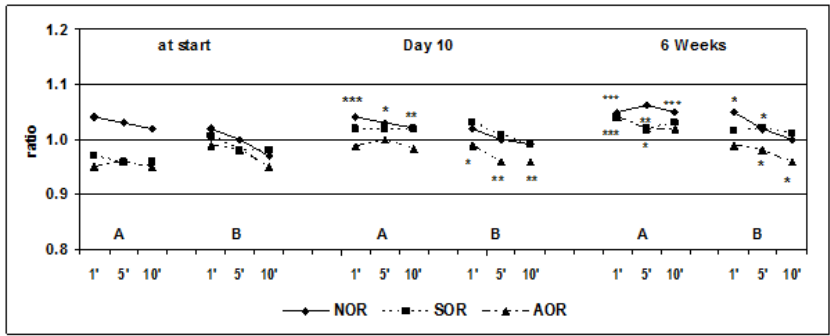

Figure 1: Changes in the mean values of the HR (a) and SBP (b) during the active orthostatic test, given as a ratio of the outcome and the baseline values; $A=$ active standing; $B=$ lying-position. ${ }^{*} p<0.05,{ }^{* *} p<0.01,{ }^{* * *} p<0.001=$ significant difference compared to the baseline values.

The role of the muscle pump in the pathogenesis of orthostatic dysregulation in patients with peripheral nervous system diseases is well known. Our study shows that after the course of PT later continued as at home exercise regimen, a normalization of the orthostatic reactivity in the patients with DNP and the pathological orthostatic autoregulation was seen. It was still pronounced at 6 weeks from the start of the treatment. These findings were associated with an improvement of the muscle strength and the muscle mass of the calves in all patients with DNP which resulted in better muscle pump efficacy.

The beneficial effect of the PT on orthostatic autoregulation, observed in our study, is probably due to various mechanisms. The Yamomoto et al. [10] have revealed that the physical exercises stimulate the proprioceptive information connected with a static posture and the motor human activity, implemented through the motoric-visceral reflexes. It is well known that the moderate to intensive exercise stimulates the sympathic nervous system, whereas in the recovery period after physical loading the parasympathic activity is dominating. Thus, during the orthostasis in healthy subjects the normal autonomic response provides adaptive changes in the systemic hemodynamics that keep the cerebral circulation stable and prevent from orthostatic hypotension [10-13].

The patients with DNP had a limited motor activity, known to be associated with a decrease physical working capacity, a decreased muscle power, deteriorated nitrogen and protein balance, cardiovascular disturbances and depression $[14,15]$. In such patients, the appropriate physical exercise can prevent not only the hypokinetic syndrome, but also can prevent the development of clinical complications by improving the glucose control [16-18], the lipid profile [19], via a normalization of the blood pressure [20, 21] and the restoration of the physical [22] and the mental health [23]. It has been demonstrated that in patients with DNP the exercises, including frequent change on postural body position, stimulate the adaptation of the systemic haemodynamics to orthostatic stimuli mainly through the improvement of the cardiovascular response [8].

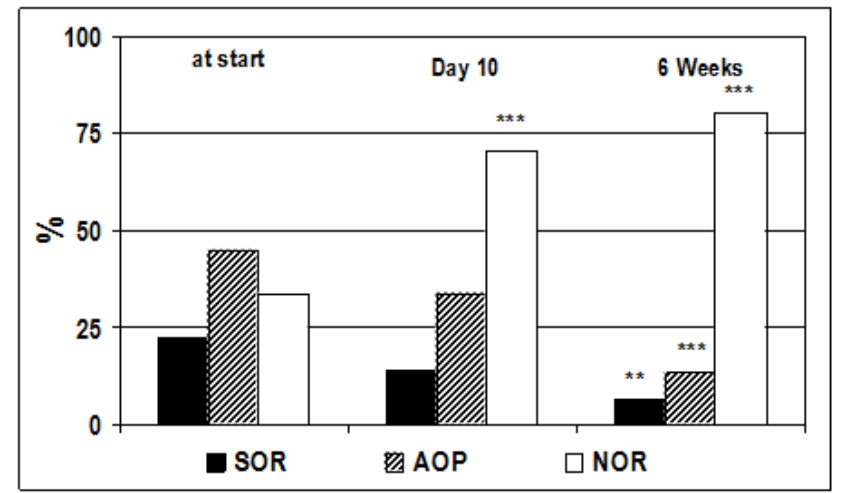

Figure 2: Percent distribution of the patients according to the type of the orthostatic reactivity at start, at day 10 and 6 weeks after the PT. * $p<0.05,{ }^{* *} p<0.01,{ }^{* * *} p<0.001=$ significant difference compared to the percent distribution of the patients before the treatment.

The short-term treatment with intravenous infusion of alpha-lipoic acid also played role in the clinical improvement of the treated DNP patients. Previous investigations have shown that a monotherapy with alpha-lipoic acid tends to improve the orthostatic dysregulation in diabetic patients but the results were not statistically significant $[3,24]$. Our study reveals that when this treatment is combined with a specialized PT, a significant improvement of the orthostatic reactivity in the patients with DNP was observed, which support the simultaneous administration of both therapeutic approaches.

In conclusion, the current study demonstrates that the structured PT, combined with 7-10 days alpha-lipoic acid treatment and continued as a home exercise programme significantly improves the orthostatic reactivity in patients with orthostatic dysregulation due to DNP. 


\section{Acknowledgment}

None of the authors or their institution has received any commercial financial support related to this study. This work was partly supported from the National Sports Academy “V. Levski” - Sofia, Bulgaria.

\section{References}

1. Low PA, Benrud-Larson LM, Sletten DM, Opfer-Gehrking TL, Weigand SD, O'Brien PC, Suarez GA, Dyck PJ. Autonomic symptoms and diabetic neuropathy: a population-based study. Diabetes Care. 2004;27(12):2942-7.

2. Maser RE, Lenhard MJ. Cardiovascular autonomic neuropathy due to diabetes mellitus: clinical manifestations, consequences, and treatment. J Clin Endocrinol Metab. 2005;90(10):5896-903.

3. Velcheva P, Damianov N, Dimitrov E, Titianova K, Hristova T, Strahilova, Karakoleva Y. Cardiovascular autonomic disturbances in diabetic polyneuropathy: therapeutic effect of the alfa-lipoic acid. Bulgarian Neurology. 2004; (4): 178-181.

4. Wagner E. Fortgesetzte Untersuchungen uber den einfluss der schwere auf den kreislauf. Arch. Ges. Physiol. 1886; (39): 371.

5. Titianova E, Nader H. Gravity and peripheral muscle pump on the cerebral orthostatic autoregulationin healthy subjects: normal values. Neurologia Balkanica. 1999; (3): 30-40.

6. Petkov I, Nenkova S. Clinical and functional rehabilitation after veloergometric exercising of elderly patients with diabetic polyneuropathy. Sport and Science. 1997; (1): 63-69.

7. Thulesius $\mathrm{O}$. Pathophysiological classification and diagnosis of hypotension. Cardiology. 1976; (1): 180-190.

8. Grahan C, Losko M, Carthey P. Exercise option for persons with diabetes complication. Diabetes Educ. 1990; (16): 212220.

9. Mueller M, Kwon O. Walking pattern used to reduce forefoot plantar pressures in people with diabetes neuropathies. Physical Therapy. 2001; (81): 828-835

10. Yamamoto Y, Hughson R, Peterson J. Autonomic control of heart rate during exercise studies by heart rate variability spectral analisis. J Appl Physiol. 1991; (71): 1136-1142.

11. Pott $J$, Raven P. Effect of dynamic exercise on human carotid cardiac baroreflex latency. Am J Physiol. 1995; (268): 12081214.

12. Pott J, Shi X, Raven P. Carotid baroreflex responsiveness during dynamic exercise in humans. Am J Physiol. 1993; (65): 1928-1938.

13. Valbon C. Badily responses to immobilization. Krusen handbook of physical medicine and rehabilitation. 1982: (3): 963-975.

14. Scheider S. Long-term exercise programs: The health professional's guide to diabetes and exercise. N. Ruderman and J. Devlin. Eds Alexandria. American Diabetes Association, 1995: 125-132.

15. Vinik A. Neuropathy. In N. Ruderman and J. Devlin. The health professional's guide to diabetes and exercise. Eds Alexandria. American Diabetes Association, 1995: 183-197.

16. Horton E. Exercise and physical training: effects on insulin sensitivity and glucose metabolism. Diabetes Metab Rev. 1986; (2): 1-17.

17. Koivisto $\mathrm{V}$, Yki-Jarvinen $\mathrm{H}$, DeFronzo R. Physical training and insulin sensitivity. Diabetes Metab Rev. 1986; (1): 445-481.

18. Mayer-Davis E, D'Agostino R, Karta A, Haffner S, Rewers M,
Saad M, R. Bergman R. Intensity and amount of physical activity in relation to insulin sensitivity. JAMA. 1998; (279): 669-674.

19. Kohl H, Gordon N, Villegas J, Blair S. Cardiorespiratory fitness, glycemic status, and mortality risk in men. Diabetes Care. 1992; (15): 185-192.

20. Krotkiewski M, Lonnroth P, Mandroukas K, Wroblewski G. Zand Rebuffe-Scrive, The effects of physical training on insulin secretion and effectiveness and on glucose metabolism in obesity and type 2 (non insulin dependent) diabetes mellitus. Diabetologia. 1985; (28): 881-890.

21. Schneider $S$, Khachadurian $A$, Amorosa $L$, Clemow $L$ Ruderman N. Ten-year experience with an exercise-based outpatient lifestyle modification program in the treatment of diabetes mellitus. Diabetes Care. 1992; (15): 1800-1810.

22. Wing R. Behavioral strategies for weight reduction in obese type 2 diabetic patients. Diabetes Care. 1989; (12): 139-144.

23. Vasterling J, Sementilli M, Burish T. The role of aerobic exercise in reducing stress in diabetic patients. Diabetes Educ. 1988; (14): 197-201.

24. Ziegler D. Effects of treatment with antioxidant alpha-lipoic acid cardiac autonomic neuropathy in NIDDM, patients: a 4month random. Controlled multicenter trial |DEKAN| Study. Diabetes Care. 1997; (20): 367-373. 